The Editor BMJ

BMA House, Tavistock Square

London WC1H 9JR

editor@bmj.com

$\mathrm{T}+44(0) 2073874499$

$\mathrm{F}+44(0) 2073836418$

BMA MEMBERS' INQUIRIES membership@bma.org.uk 02073836599

BMJ CAREERS ADVERTISING sales@bmjcareers.com 02073836531

DisPlay ADVERTISING

sales@bmj.com

02073836350

REPRINTS

reprints@bmj.com

02083461339

SUBSCRIPTIONS

subscriptions@bmj.com

02073836270

For "Who is Who" at the BMJ

see bmj.com/contacts

For advice to contributors see bmj.com/advice

To submit an article go to submit.bmj.com

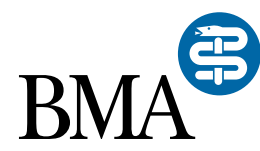

The $B M J$ is published by BMJ Publishing Group Ltd, a wholly owned subsidiary of the British Medical subsidiary of theciation.
Assol

The BMA grants editorial freedom to the Editor of the BMJ. The views expressed in the journal are those of the authors and may not necessarily comply with BMA policy.

The BMJ follows guidelines on editorial independence produced by the World Association of Medical Editors (www.wame.org/wamestmt.htm\# independence) and the code on good publication practice produced by the Committee on Publication Ethics (wwwipublicationericsorger cope1999/gpp/gpp.phtml\#gpp).
(CBMJ Publishing Group Ltd 2005.

All Rights Reserved. No part of this publication may be reproduced, stored in a retrieval system, or transmitted in any form or by any other means, electronic, mechanical, photocopying, recording, or otherwise, without prior permission, in writing, of the BMJ

US second class postage paid at Rahway, NJ. Postmaster: send addres changes to BMJ, c/o Mercury Airfreight International Ltd Inc, 365 Blair Road, Avenel, NJ 07001 , USA. $\$ 640$. Weekly

Printed by Wyndeham Heron and Co

\section{This week in the BMJ}

Demedicalising
neck pain helps
patients

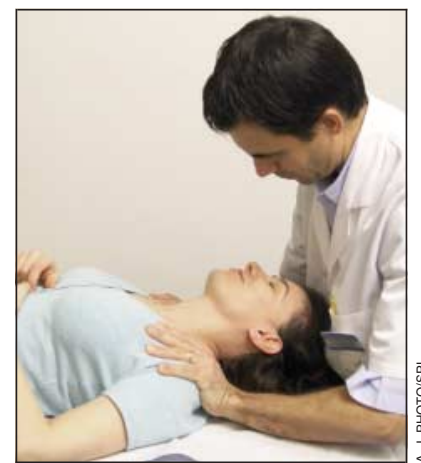

Brief physiotherapy interventions focused on demedicalising the problem and teaching the principles of cognitive behaviour therapy can be as beneficial as standard physiotherapy for patients who prefer this treatment. Klaber Moffett and colleagues (p 75) randomised 268 patients to standard physiotherapy (five sessions) or up to three sessions of the brief intervention. Overall, patients who received standard care reported a significant improvement compared with patients in the brief intervention group, but patients who preferred the brief intervention and received this treatment had similar outcomes to patients receiving usual physiotherapy.

\section{Expertise based} trials may be the way forward for evaluating surgical interventions

Although a randomised controlled trial is the best study design for evaluating pharmacological interventions, scepticism about whether it is best in non-pharmacological interventions (such as surgery) remains. Devereaux and colleagues (p 88) propose an alternative: the expertise based randomised controlled trial, in which participants are randomised to clinicians with expertise in intervention $\mathrm{A}$ or intervention $\mathrm{B}$. They argue that increased use of the expertise based design will enhance the validity, applicability, feasibility, and ethical integrity of randomised controlled trials in surgery and other non-pharmacological areas.

Migraine may be a risk factor for stroke

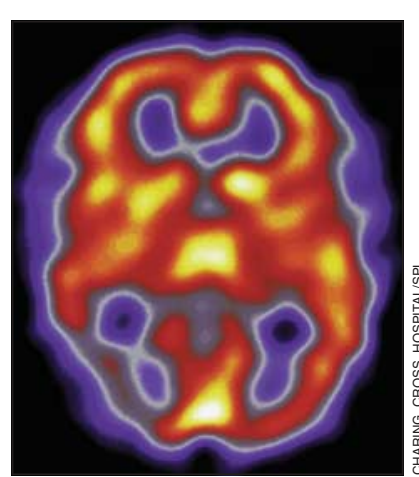

Migraine may be an independent risk factor for ischaemic stroke, and the risk is higher among oral contraceptive users and younger adults. Pooling the data from 11 case-control studies and three cohort studies, Etminan and colleagues ( $p$ 63) found that having migraines more than doubles the risk for an ischaemic stroke (relative risk $2.16,95 \%$ confidence interval 1.89 to 2.48 ). The risk was greater in people who had migraine with aura than in those without aura, and was about eightfold greater in users of oral contraceptives than in those not using them.

\section{Trials of gene} transfer in humans need more caution

The public, rather than trial participants, will likely bear many of the consequences of latent risks associated page 79, Kimmelman reviews recent developments in gene transfer in humans, focusing on the risks in such clinical trials, especially for healthy trial participants. He argues that uncertainties of risks demand central protocols and emphasises that so far only the United Kingdom and Australia have implemented measures to track the health of trial participants in the long term. with gene transfer. On ethical review of all trial

\section{Decision makers need to judge credibility of economic evaluations}

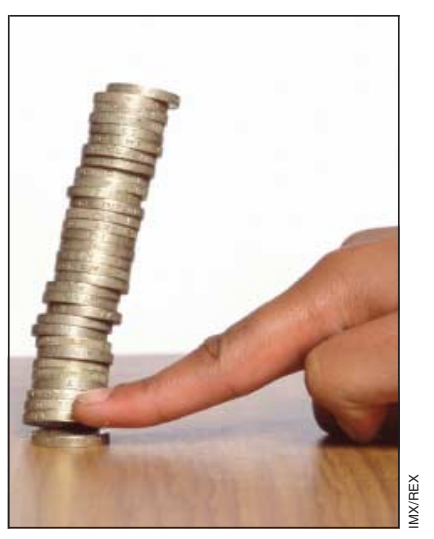

Cost effectiveness assessments submitted to the technology appraisal programme of the National Institute for Clinical Excellence (NICE) by manufacturers of the relevant healthcare technologies differ significantly from those contracted by university based assessment groups. Miners and colleagues ( $\mathrm{p}$ 65) retrospectively compared 62 appraisals. Manufacturers' estimates of incremental cost effectiveness ratios were lower (suggesting a more cost effective use of resources) than those produced by the assessment groups (25 were lower, 29 were the same, none were higher; $\mathrm{P}<0.01)$. 


\section{POEM* \\ Optimal oral antiplatelet therapy for vascular disease}

Question Which antiplatelet agents, used alone or in combination, are effective in preventing recurrent vascular events?

Synopsis Aspirin prevents recurrent vascular events in a wide range of high risk patients, but it is unknown if other antiplatelet agents, such as clopidogrel or dipyridamole, alone or in combination with aspirin, are more effective. The investigators rigorously searched multiple databases including Medline, the Cochrane clinical trials registry, and reference lists of trials, review articles, and scientific statements and guidelines of official societies. They included randomised trials comparing an antiplatelet regimen to either placebo or another antiplatelet regimen assessing outcomes for at least 10 days. They identified 111 trials enrolling nearly 100000 patients. The investigators do not state if the search for, and evaluation of, the included studies was done independently by more than one person. No formal assessment of the potential for publication bias was done, nor was any specific analysis done to determine homogeneity of the results. Recommended oral first line antiplatelet therapy is aspirin for patients with ST segment elevation myocardial infarction; aspirin or clopidogrel for those with initial transient ischaemic attack (TIA) or ischaemic stroke, chronic stable angina, or peripheral arterial disease (since aspirin is less expensive, clopidogrel should be reserved only for patients who do not tolerate aspirin); and aspirin plus clopidogrel for those with non-ST segment elevation acute coronary syndrome. For second line therapy, the combination of aspirin and clopidogrel is recommended for recurrent acute coronary syndrome. The combination of aspirin and clopidogrel does not, however, lower the incidence of recurrent vascular events in patients with recurrent TIA or ischaemic stroke, but does increase the risk of major and life threatening bleeding. The combination of aspirin and extended release dipyridamole is therefore recommended for patients with recurrent TIA or ischaemic stroke in the absence of known coronary artery disease. Because of the theoretical risk of dipyridamole exacerbating myocardial ischaemia, further studies are needed before firm recommendations can be made on the management of patients with both recurrent TIA or ischaemic stroke and known coronary artery disease. Ticlopidine is beneficial for various vascular conditions, but common side effects-some serious-limit its usefulness.

Bottom line Aspirin is the recommended oral first line antiplatelet therapy for patients with ST segment elevation myocardial infarction. Aspirin or clopidogrel is recommended for those with initial TIA or ischaemic stroke, chronic stable angina, or peripheral arterial disease, and aspirin plus clopidogrel should be used for those with non-ST segment elevation acute coronary syndrome. For second line therapy, the combination of aspirin and clopidogrel is recommended for recurrent acute coronary syndrome. The combination of aspirin and extended release dipyridamole is recommended for patients with recurrent TIA or ischaemic stroke in the absence of known coronary artery disease.

Level of evidence la (see www.infopoems.com/levels.html). Systematic review of randomised trials displaying worrisome heterogeneity.

Tran H, Anand SS. Oral antiplatelet therapy in cerebrovascular disease, coronary artery disease, and peripheral arterial disease. JAMA 2004;292:1867-74

CinfoPOEMs 1992-2004 www.infoPOEMs.com/informationmastery.cfm

* Patient-Oriented Evidence that Matters. See editorial (BMJ 2002;325:983)

\section{Editor's choice}

\section{Death by tsunami and poverty}

Fishermen in the Indian Ocean were unaware of the wave that swept past them and devastated their homes and families. How many of us are unaware that over a million people died in the 1970 s after a cyclone struck Bangladesh and an earthquake shook China? What, then, makes this tsunami disaster so potent-beyond unprecedented television and newspaper coveragethat the United Nations will have launched a flash appeal by the time you read this week's $B M J$ ?

A wave that killed on two continents is a stark reminder that the many divisions within and between nations are insignificant when compared with the challenge posed by nature. Increasingly, too, we live in an interconnected and interdependent world. Strengthening infrastructure and the potential for medical response in poorer countries garners moral support among people in rich countries and also helps all citizens of our world. Among the dead or missing after this Asian catastrophe are more than 5000 Europeans-a small number in global terms, but a major disaster for Europe.

Kofi Annan has described this tsunami as the worst natural disaster his organisation has faced. The death toll is 150000 and rising. The World Health Organization believes the grieving of millions will be compounded by waterborne diseases related to unsafe drinking water and inadequate sanitation (p 59). How much of the billion or so pounds of aid already pledged will materialise? How much will be rendered ineffectual by the threadbare infrastructures of many of the affected countries? How do we conjure clean water, roads, and health services from empathy?

As charities and rock stars join forces in a campaign to make poverty history (www.makepovertyhistory.com), the British government has a pivotal role to play in its presidency of the G8-a policy group of the world's eight most powerful nations-and through its presidency of the European Union (p 59). "We must now all accept the utter futility of trying to shut up our borders to problems abroad," says Prime Minister Tony Blair in last week's Economist, where he states his two priorities as alleviating poverty in Africa and combating climate change. The challenge for him, though, is to magic real change from these admirable intentions.

Our admirable intentions to keep bmj.com free flounder this week. We begin charging for the content published from the beginning of 2005, as explained in an editorial last October (BMJ 2004;329:814). Each issue will be free for the first week after publication; content will be behind access controls for the next 51 weeks, then free again. Research papers and many of the services on our website will remain free throughout. In addition, BMA members, personal subscribers, and people from HINARI's list of resource poor countries will continue to receive full free access. Other readers can buy an annual subscription or pay per article. We will keep this charging policy under constant review.

Kamran Abbasi acting editor (kabbasi@bmj.com)

To receive Editor's choice by email each week subscribe via our website: bmj.com/cgi/customalert 